\title{
Neural Mechanisms of Anger Regulation as a Function of Genetic Risk for Violence
}

\author{
Nelly Alia-Klein, Rita Z. Goldstein, \\ Dardo Tomasi, and Patricia A. Woicik \\ Brookhaven National Laboratory \\ Benjamin Williams and Ian W. Craig \\ Kings College, London, England
Gene-Jack Wang and Joanna S. Fowler
Brookhaven National Laboratory and Mount Sinai School of Medicine

\author{
Scott J. Moeller \\ University of Michigan, Ann Arbor \\ Frank Telang and Anat Biegon \\ Brookhaven National Laboratory \\ Nora D. Volkow \\ National Institute on Drug Abuse
}

\begin{abstract}
Genetic risk may predispose individuals to compromised anger regulation, potentially through modulation of brain responses to emotionally evocative stimuli. Emphatically expressed, the emotional word No can prohibit behavior through conditioning. In a recent functional magnetic resonance imaging study, the authors showed that healthy males attribute negative valence to No while showing a lateral orbitofrontal response that correlated with their self-reported anger control. Here, the authors examined the influence of the monoamine oxidase A (MAOA) gene (low vs. high transcription variants) on brain response to No and in relationship to trait anger reactivity and control. The orbitofrontal response did not differ as a function of the genotype. Instead, carriers of the low-MAOA genotype had reduced left middle frontal gyrus activation to No compared with the high variant. Furthermore, only for carriers of theup low-MAOA genotype, left amygdala and posterior thalamic activation to No increased with anger reactivity. Thus, vulnerability to aggression in carriers of the low-MAOA genotype is supported by decreased middle frontal response to No and the unique amygdala/thalamus association pattern in this group with anger reactivity but not anger control.
\end{abstract}

Keywords: MAOA, emotion regulation, anger, response to no, fMRI

Supplemental materials: http://dx.doi.org/10.1378/a0015904.supp

To understand complex phenotypes, researchers have studied negative emotions and their inhibitory control; and among the negative emotions, there is a growing recent interest in emotional reactivity and anger control (Hewig, Hagemann, Seifert, Naumann, \& Bartussek, 2004). Indeed, violent behavior is a public health problem, and the experience and the outward control of

Nelly Alia-Klein, Rita Z. Goldstein, Dardo Tomasi, Patricia A. Woicik, Frank Telang, and Anat Biegon, Medical Department, Brookhaven National Laboratory, Upton, NY; Scott J. Moeller, Department of Psychology, University of Michigan, Ann Arbor; Benjamin Williams and Ian W. Craig, Kings College, London, England; Gene-Jack Wang and Joanna S. Fowler, Medical Department, Brookhaven National Laboratory; and Mount Sinai School of Medicine; Nora D. Volkow, National Institute on Drug Abuse, Bethesda, MD.

This research was conducted at Brookhaven National Laboratory under Contract DE-AC-298CH10886 with the U.S. Department of Energy, and it was supported by its Office of Biological and Environmental Research, the National Institutes of Health General Clinical Research Center (Grant No. MO1RR10710), the National Institute on Drug Abuse (Grant No. K05DA020001), and the National Association for Research on Schizophrenia and Depression.

Correspondence concerning this article should be addressed to Nelly Alia-Klein, Medical Department, Brookhaven National Laboratory, P. O. Box 5000, Upton, NY 11973-5000. E-mail: nellyklein@bnl.gov anger may contribute to the propensity for violent behavior ( $\mathrm{Da}$ vidson, Putnam, \& Larson, 2000; Harmon-Jones, 2004; Driscoll, Zinkivskay, Evans, \& Campbell, 2006). Anger is a psychobiological emotional state that varies among individuals (Dimberg, 1986), but with recurrent social conflict it may develop into stable trait patterns (Spielberger, 1988). Previous neuroimaging studies show that anger-related stimuli engage corticolimbic neural circuitry of high-arousal negative emotions (e.g., regions of the prefrontal cortex, amygdala, hippocampus, insula, and thalamus; Murphy, Nimmo-Smith, \& Lawrence, 2003). Anger reactivity and control involve high-arousal emotions that influence affect regulation and are related to the emergence of behavioral self-control (Barratt, Stanford, Dowdy, Liebman, \& Kent, 1999). In turn, the development of self-control is possibly related to the broader construct of conscience as expressed in social situations that challenge goal-directed behavior. Thus, anger regulation consists of the interplay between cognitive, affective, and relational processes that influence how humans may construct the meaning of relational words. The word No, whether heard or expressed, is a relational word, and it is possibly one of the most potent in challenging goal-directed behavior (Gopnik, 1984).

Much of goal-directed behavior is constrained by rules and prohibitions imposed from the rearing environment and later in- 
ternalized during development (Kochanska \& Knaack, 2003). During early language development, relational words are words of primary social use (Bloom, 1973): The utterance No, for example, is used in a developmentally predictable manner in a relational context, first to reject and then to refuse or protest. Later in development, as higher-order executive functions develop, No is used to comment on one's own plan (Gopnik \& Meltzoff, 1985). In adulthood, No is expressed in varied situations and connotations; the utterance $N o$ ! in a relational context can be used to caution and thus promote safety, to refuse to engage in unwanted activities (e.g., to reject sexual advances), and/or to express disagreement (e.g., "No, I don't agree with you").

In our previous functional MRI (fMRI) study, we documented behavioral and blood oxygen level-dependent (BOLD) responses to hearing and reiterating the verbal utterance No emphatically expressed and its BOLD correlation with trait anger control in healthy men (Alia-Klein et al., 2007). We found that, in the absence of contextual cues, hearing No emphatically expressed is rated as negatively valenced, compared with another word $(U p)$ that was matched on frequency in the English language and on the tone of utterance. It is interesting that, when probed to write a phrase on their thoughts upon hearing No, participants had the following associations: "Mom yelling at me as a kid," "to discipline someone harshly," "dog getting yelled at," "bad day," "don't do this," "I did something wrong" "no means no," and so forth. During the task, participants were instructed to press a button immediately upon detection of $N o$ or a control word $(U p)$; and significantly slower response times were produced in response to No, which was interpreted as behavioral conflict.

The fMRI BOLD results implicated the right lateral orbitofrontal cortex (OFC), a prefrontal region associated with inhibitory control in the responses to No. Furthermore, the observed OFC activation to No was modulated by individual differences in trait levels of anger control. In our interpretation of this finding, we reasoned that participants who attended to $\mathrm{No}$ as a valenced signal were also better adept at inhibiting negative emotion, allowing those reporting high anger control to cease behavior (as in hearing No or saying No to oneself and withdrawing one's ongoing motor response; Alia-Klein et al., 2007).

In this follow-up study, we explored correlations between trait anger reactivity and control (i.e., anger regulation) and neural response to No. In addition, here for the first time, we examined the role of genetic predisposition in neural response to No and in anger regulation. Because propensity toward anger is associated with reactive aggressive or violent behavior (Scarpa \& Raine, 1997), and the latter is at least partly genetically mediated (Eisenberger, Way, Taylor, Welch, \& Lieberman, 2007; MeyerLindenberg et al., 2006), our present focus was on anger regulation traits as a function of genetic risk for violent behavior.

In particular, we focused on the genetic variability specifically identified in the X-linked MAOA gene (MAOA, MIM 309850; Sabol, Hu, \& Hamer, 1998) that has been previously associated with propensity or risk to perpetrate violence. The gene product of MAOA, monoamine oxidase A (MAO A), regulates the enzymatic metabolism of neurotransmitters serotonin, dopamine, and norepinephrine, thereby modulating emotion regulation and general brain function (Fowler et al., 1987; Shih, Chen, \& Ridd, 1999). In humans and primates, categorization of common genetic variability (vs. a rare mutation) is based on a functional polymorphism in the promoter region of the MAOA gene; a variable number tandem repeat (VNTR; 3.5 or 4 repeats [i.e., high] and 2, 3, or 5 [low] repeats) is common in the male population and occurs in a $\sim 60: 40$ ratio in humans. As compared with the high variant, the low variant has relatively lower transcriptional activity in human nonneural cell lines (Sabol et al., 1998) and is associated with aggressive and violent behavior when combined with childhood maltreatment (Foley et al., 2004; Kim-Cohen et al., 2006). Some studies have also reported main effects of the genotype on impulsive aggressive behavior (Jacob et al., 2005; Manuck, Flory, Ferrell, Mann, \& Muldoon, 2000). We have recently reported that the high- and low-MAOA genotype do not differ with respect to brain MAO A levels (Fowler et al., 2007) and that in vivo MAO A levels (but not the MAOA genotype) predicts trait aggression (AliaKlein, Goldstein, et al., 2008).

However, with a growing number of fMRI studies of healthy participants who differ in their MAOA genotype, neural mechanisms have been revealed; these neuroimaging studies propose that functional neuroanatomy is an endophenotype (Gottesman \& Gould, 2003) or a mechanism intermediate to the phenotype (for a review, see Meyer-Lindenberg \& Weinberger, 2006). Specifically, relevant intermediate phenotypes in key corticolimbic regions in response to emotional and cognitive tasks significantly differed as a function of the MAOA genotype (Eisenberger et al., 2007; Fan, Flombaum, McCandliss, Thomas, \& Posner, 2003; Fan, Fossella, Sommer, Wu, \& Posner, 2003; Passamonti et al., 2006). Of particular relevance is one comprehensive study in which individuals with the low-MAOA genotype showed differential activity in amygdala, hippocampus, and insula, among other regions, in response to paradigms of negative emotion (Meyer-Lindenberg et al., 2006).

Here we build on these recent studies to examine brain and behavior responses to the No task in a group of healthy nonsmoking males who also completed a trait anger questionnaire and were stratified by their MAOA genotype. We predicted that lateral OFC responses to No (Alia-Klein et al., 2007) would be reduced in the low-MAOA group. In addition, we predicted that individual differences in trait anger regulation will have a measurable influence on neural response to $\mathrm{No}$ in corticolimbic regions and that these effects will differ as a function of the MAOA genotype.

\section{Method}

\section{Participants}

Twenty-seven nonsmoking males participated in this study. Data for 23 of these participants were reported previously (AliaKlein et al., 2007). Here, we also performed genetic analyses in all 27 participants, which resulted in 13 participants classified as having low-MAOA repeat alleles and 14 as having high-MAOA repeat alleles. There were no differences between these genotype groups in age, education, right-hand dominance (Oldfield, 1971), socioeconomic status (Hollingshead, 1975), measures of general intellectual functioning (verbal intelligence as estimated with the Reading subscale of the Wide Range Achievement Test [3rd ed.; Wilkinson, 1993] and nonverbal intelligence as estimated with the Matrix Reasoning subset of the Wechsler Abbreviated Scale of Intelligence [Wechsler, 1999]), self-reported depression (Beck, Steer, \& Brown, 1996), or anger (Spielberger, 1988; see Table 1). 
Table 1

No Differences Between the Low-and High-MAOA Genotype Groups on Demographic Characteristics and Anger Regulation

\begin{tabular}{lccc}
\hline & \multicolumn{3}{c}{$M \pm S D$ for: } \\
\cline { 2 - 3 } \multicolumn{1}{c}{ Demographic } & $\begin{array}{c}\text { Low MAOA } \\
(n=13)\end{array}$ & $\begin{array}{c}\text { High MAOA } \\
(n=14)\end{array}$ & $p$ \\
\hline Age (years) & $31.6 \pm 1.7$ & $29.2 \pm 1.3$ & .30 \\
Education (years) & $15.2 \pm 0.4$ & $15.1 \pm 0.7$ & .99 \\
Laterality quotient & $0.82 \pm 0.1$ & $0.96 \pm 0.01$ & .30 \\
Socioeconomic status & $42.6 \pm 3.0$ & $41.1 \pm 3.6$ & .75 \\
Intelligence & & & \\
$\quad$ Matrix-scaled score & $12.0 \pm 0.5$ & $12.2 \pm 0.3$ & .75 \\
$\quad$ WRAT-3-scaled score & $104.2 \pm 3.8$ & $103.1 \pm 3.8$ & .83 \\
Depression in past 2 weeks & $4.9 \pm 2.0$ & $6.5 \pm 2.1$ & .60 \\
Anger reaction & $47.5 \pm 3.5$ & $43.1 \pm 3.1$ & .37 \\
Anger control & $51.8 \pm 2.6$ & $55.9 \pm 2.3$ & .27 \\
\hline
\end{tabular}

Note. Trait scores on the revised State-Trait Anger Expression Inventory for anger reaction and anger control were converted to $t$ scores: Anger reactivity scores ranged from 28 (less reactivity) to 72 (more reactivity); anger control scores ranged from 36 (less control) to 68 (most control). MAOA $=$ monoamine oxidase A; Matrix $=$ Matrix Reasoning subset of the Wechsler Abbreviated Scale of Intelligence; WRAT-3 = Wide Range Achievement Test (3rd ed.).

Individuals were excluded for current or past psychiatric or neurological disease, history of drug or alcohol abuse or dependence including cigarette smoking (tested by self-report and breath carbon monoxide test), positive urine screen for drugs of abuse, history of head trauma with loss of consciousness, history of cardiovascular or endocrinological disease, current medical illness, and contraindication to MRI (e.g., having metallic implants in the body or claustrophobia). Participants were fully informed of the nature of the research and provided written consent for their involvement in this study in accordance with the local institutional review board.

\section{Genotype Analysis}

DNA for MAOA genotyping was obtained from cheek swab samples from each participant (Freeman et al., 2003). The polymerase chain reactions were performed as previously described by (Sabol et al., 1998) and analyzed on an Applied Biosystems 3100 Genetic analyzer. Alleles were observed in expected ranges using Genescan, Version 3.7, and Genotyper software, Version 3.6. As expected, all participants in this study had either the low-MAOA genotype (i.e., 3 repeats) or the high-MAOA genotype (4 repeats). According to Sabol et al. (1998), 3 and 4 repeats are the most common alleles in the general population $(35.6 \%$ and $62.4 \%$ respectively) with a much lower prevalence of individuals with 3.5 -repeats allele $(0.4 \%$ in the population) and 5 repeats $(1.4 \%)$.

\section{Trait Anger}

Several hours before the fMRI scan, all participants completed the revised State-Trait Anger Expression Inventory (STAXI-2), a 57-item questionnaire that assesses trait anger, defined as a longstanding emotional bias toward the experience and the expression of anger (Spielberger, 1988). The STAXI-2 has 12 scales/ subscales; of these, we focused on the Angry Reaction scale, which measures anger as a reaction to criticism, perceived affronts, and negative evaluation by others, and the Anger Control-Out (AC-O) scale, which measures how often a person controls the outward expression of their anger. Examples of Angry Reaction items on the STAXI-2 include, "I get angry when slowed down by others," and "It makes me furious when I'm criticized in front of others." Examples of Anger Control items include, "I can stop myself from losing my temper," and "I control my angry feelings." In the present sample, scores on both measures fell within the normative range (see Table 1), which is based on a sample of more than 600 healthy men. Alpha coefficients of this normative sample ranged from 0.73 to 0.91 for the STAXI-2 subscales (Spielberger, 1988). In this healthy sample, trait anger reactivity and control measures were negatively correlated ( $n=27, r=-0.38, p=.03$ ). Thus, the higher the self-reported anger reactivity, the lower the anger control across all study participants.

\section{fMRI Task}

The No task was described in detail previously (Alia-Klein, Goldstein, et al., 2007). In brief, the task involved emphatic vocalizations with simultaneous visual display of four word stimuli (No, Up, Yes, Ten) each presented for $2 \mathrm{~s}$ alternating with a 2-s fixation cross in a block design. Each of a total of four task runs comprised eight pseudorandomized 18-s word blocks (four of the same word in each block) resulting in a total of 36 presentations per word. The word conditions alternated with 18-s fixation baseline without auditory or visual stimulation. Throughout this fMRI task, participants were instructed to "subvocally express the word" and "respond by pressing the button as soon as you see and hear a word," as previously described (Alia-Klein et al., 2007). Reaction time (RT; the time from word presentation to button press) and performance accuracy (percentage of button presses within the 2,000-ms allowable time window) were obtained throughout this fMRI task (except for 2 participants who did not have RT data because of technical problems). These measures were averaged across all runs, separately within each word condition. Immediately after fMRI, participants provided ratings of the words on valence (positive vs. negative), intensity, and interest. In the present study, we examined results specifically for $N o$ and $U p$, which were matched on number of letters, frequency of use in the English language (Kučera \& Francis, 1967), and on emphatic vocalization.

\section{Statistical Analysis of Task-Related Behavior and Self-Reported Anger}

We conducted a 2 (word condition: No, Up) $\times 2$ (MAOA genotype group: low, high) analysis of variance (ANOVA) using SPSS (SPSS Inc., Chicago) for each of the behavioral variables (RT, accuracy, valence, intensity, and interest ratings; see also Table S1 in the Supplementary Online Results). Two-tailed independent-samples $t$ tests were used to test differences between the groups on the variables in Table 1, including self-reported anger reactivity and control.

\section{MRI Acquisition and Processing}

We performed MRI acquisition on a 4-Tesla Varian/Siemens scanner with a SONATA gradient set. The BOLD responses were 
measured as a function of time using a T2-weighted single-shot gradient-echo planar imaging (EPI) sequence (TE/TR [reception time/echo time $]=20 / 2,000 \mathrm{~ms}$, 4-mm slice thickness, 1-mm gap, typically 33 coronal slices, $20-\mathrm{cm}$ FOV, $64 \times 64$ matrix size, $90^{\circ}$ flip angle, 200-kHz bandwidth with ramp sampling, 4 dummy scans, $92 \mathrm{~dB}$ of sound pressure level). We performed reconstruction of EPI scans and analysis of fMRI data sets using a phase correction method (Buonocore \& Gao, 1997). The SPM2 package (Wellcome Department of Cognitive Neurology, London) was used for subsequent analyses. A six-parameter rigid body transformation (three rotations, three translations) was used for image realignment to correct for head motion. The realigned data sets were normalized to the Talairach frame (Talairach \& Tournoux, 1988) with a 12-parameter affine transformation (Ashburner, Neelin, Collins, Evans, \& Friston, 1997) and the SPM2 default settings: no weighting, cutoff $=25 \mathrm{~mm}$, medium regularization, and 16 nonlinear iterations. The normalized images were saved with a trilinear interpolation without warping, with a voxel size of $3 \times$ $3 \times 3 \mathrm{~mm}$. A bounding box was defined by the following: $\mathrm{x}=$ -78 to $78 \mathrm{~mm}, \mathrm{y}=-112$ to $76 \mathrm{~mm}$, and $\mathrm{z}=-50$ to $85 \mathrm{~mm}$. An 8-mm full-width half-maximum Gaussian kernel was used to smooth the data. A general linear model (Friston et al., 1995) and a castle design with two word conditions convolved with a canonical hemodynamic response function were used to calculate the activation maps. The time series were band-pass filtered with the hemodynamic response function as low-pass filter and a 1/560-s cutoff frequency as high-pass filter.

\section{Statistical Analysis of fMRI Data}

Using the SPM2 package, we created fixed-effects images contrasting the No, $U p$, and fixation-baseline epochs ( $N o$ fixation, $U p$ fixation and $N o-U p$ ) for each participant for each run separately and then averaged them across all four fMRI runs in each particpant using IDL software (ITT Visual Information Solutions, Boulder, $\mathrm{CO}$ ). To test the main effects of $\mathrm{No}$, a one-sample $t$ test was conducted with the contrast $N o-U p$ using all 27 participants (see Figure S1 in Supplementary Online Results). To test differences between the MAOA groups on BOLD response to No, we performed a two-sample $\mathrm{t}$ test with the same contrast ( $\mathrm{No}-\mathrm{Up}$; see Figure 1). To assess BOLD response to No in relationship to the trait measures, we performed simple regression analyses in SPM2 using anger reactivity and control, as seed values separately regressed on the differential fMRI-BOLD contrast maps (e.g., $\mathrm{No}-$ $U p$ ) for each of the genotype groups (Figures 2, 3, and 4) and also across all participants (see Figures S2, S3, and S4 in Supplementary Online Results). Tables 2 and 3 list the results showing their significance separately for the genotype groups and in all the participants. To address multiple comparisons issues, we chose to evaluate only those activations that were significant at an uncorrected threshold of $p<.005$ with a 5-voxel $\left(135 \mathrm{~mm}^{3}\right)$ extent threshold.

Small volume correction (Worsley et al., 1996) was used in specific regions of interest (ROI). The functional ROI were selected a priori from the SPM2 activation results of our previous study (Alia-Klein et al., 2007) and based on findings from the large-sample fMRI study on MAOA genotype by MeyerLindenberg et al. (2006). These regions included any of our significant SPM2 activations in the amygdala (emotional reactivity), lateral orbitofrontal cortex or other prefrontal regions (emotional control), insula (emotional awareness), hippocampus (emotional memory), and thalamus (approach emotions; Dougherty et al., 2004). In all statistical parametric mapping analyses, anatomical specificity was corroborated with a coplanar stereotaxic atlas of the human brain (Talairach \& Tournoux, 1988).

\section{Analyses With Functional ROI}

For follow-up statistical analyses and confirmation of the SPM2 voxel-based analyses, the functional ROI were extracted with an isotropic volume of 27 voxels defined at significant activation clusters in these regions (e.g., Table 2 [with a custom program
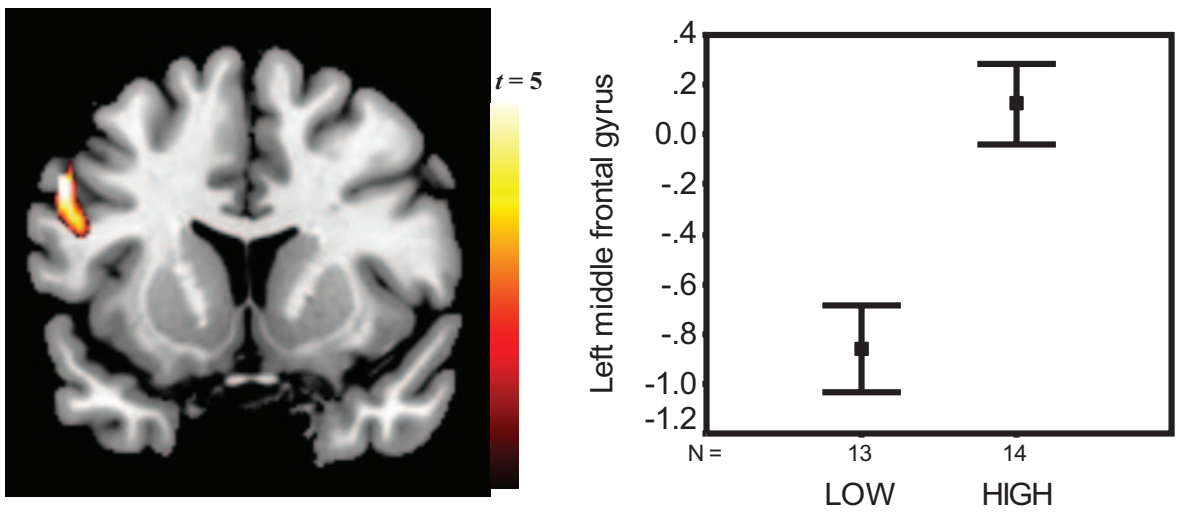

Figure 1. Gene-brain response to No. Left panel: Image of response to No as a function of the monoamine oxidase A (MAOA) genotype with corresponding standard error of the mean (SEM) bars on the right showing that low-MAOA participants had reduced percent blood-oxygen level dependent (BOLD) signal change ( $y$ axis) in left lateral middle frontal gyrus (BA 9), compared with high-MAOA participants (independent samples $t=$ $-4.1, p=.0001)$. This figure was derived from the $N o-U p$ contrast and validated through comparison of brain responses, derived from regions of interest, to No fixation and $U p$ fixation see Figure S5 in the Supplementary Online Results). 

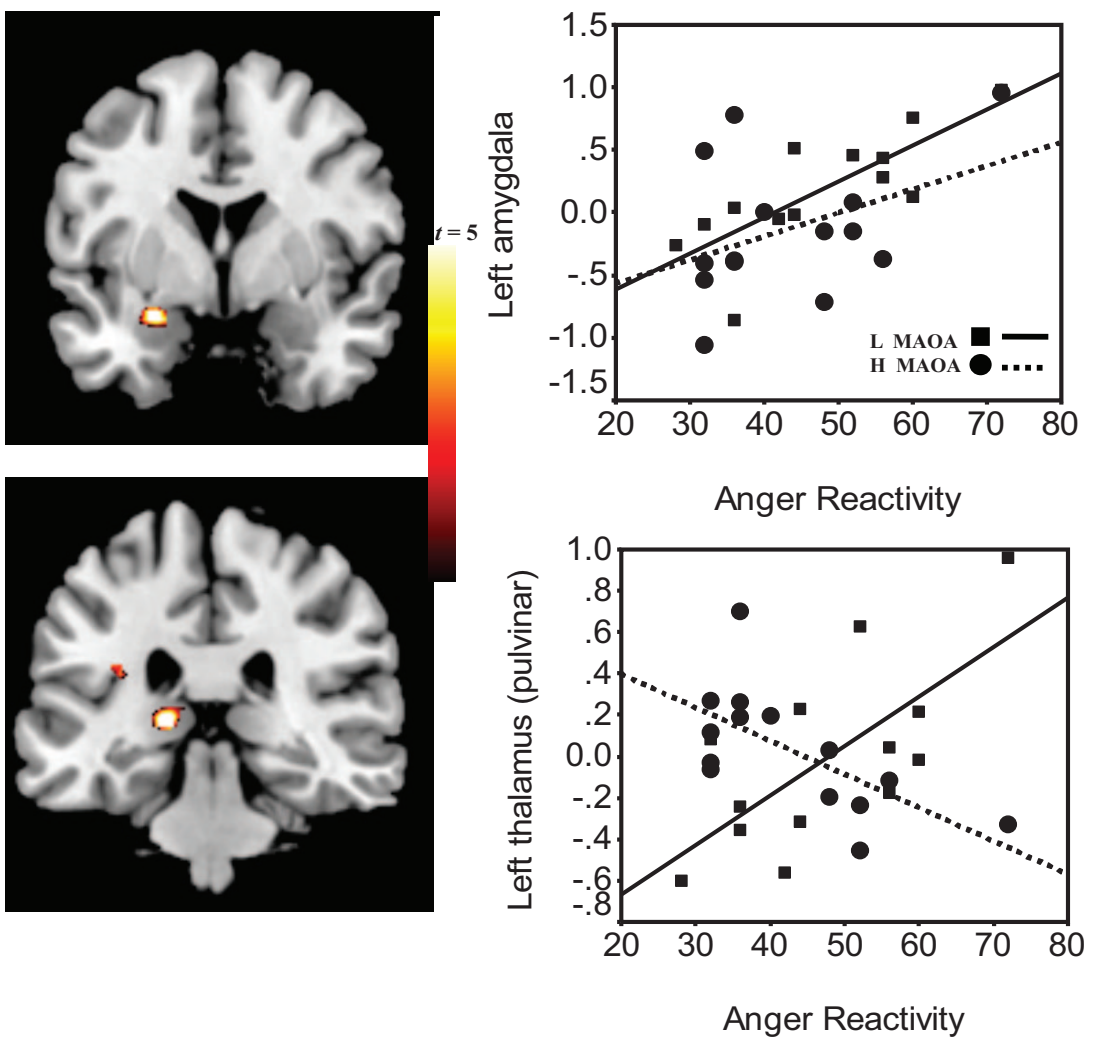

Figure 2. Gene-brain-behavior model in anger reactivity. Left panels: Images of the clusters in positive correlation with self-reported anger reactivity and blood-oxygen-level-dependent (BOLD) response to No in the left amygdala and thalamus. Right panels: Scatterplots of percent BOLD signal change to No and scores on trait anger reactivity in the left amygdala (in upper right panel: low MAOA, $R^{2}=0.61, p=.002$; vs. high, $R^{2}=0.16$, $p=.157$ ) and left thalamus (pulvinar: low, $R^{2}=0.48, p=.009$; versus high, $R^{2}=0.43, p=.011$ ). This figure was derived from the $\mathrm{No}-\mathrm{Up}$ contrast and validated through comparison of brain responses, derived from regions of interest, to No fixation and $U p$ fixation (see Table S4 in the Supplementary Online Results). Squares and solid regression line indicate low (L) MAOA; circles and dotted line indicate high (H) MAOA.

written in IDL, ITT Visual Information Solutions, Boulder, CO]) the mean (and variability) BOLD-fMRI signal amplitudes. This approach resulted in percent BOLD signal change values for each participant for each of the three contrasts (No-Up, No fixation, and $U p$ fixation). These extracted ROI were used with SPSS 11.5 (SPSS Inc., Chicago, IL; Stevens, 1992) to validate the aforementioned SPM2 analyses and to produce scatterplots.

Using the ROI values, we also tested whether the regression lines predicting trait anger were significantly different between the MAOA groups using the overall tests of coincidence ( $F$ tests; Glantz, 2005). Thus, in Tables $2-3$ and Supplementary Online Results S2-S3, correlations marked with an asterisk show significantly different correlation direction as a function of the MAOA genotype at $p<.05$. Finally, we conducted multiple regression analyses in SPSS, which allowed us to quantify the combined effect of multiple brain regions to predict trait anger (Tables 2 and 3). Statistical significance for these ROI analyses was defined as $p<.05$.

Note that our reference to $N o$ in the results is based on the contrast $N o-U p$ because it is the contrast that controls for general language and emphatic tone-related activations and captures re- sponse specifically to $N o$ and not to $U p$, which was also emphatically expressed. Nevertheless, in the Supplementary Online Results, we present analyses with $N o$ fixation and $U p$ fixation (Figure S5) and in relationship to the anger regulation measures in all participants and as a function of the MAOA genotype (Table S4 and S5) to show that the neural response to No but not to $U p$ was the one associated with the MAOA genotype effects presented in the results.

\section{Results}

\section{Behavioral Results}

There was a significant word main effect for RT, $F(1,23)=$ $5.07, p=.034$; and valence ratings, $F(1,25)=18.17, \mathrm{p}<.0001$; such that, compared with $U p$, all participants required longer time to respond to $\mathrm{No}$ and rated $\mathrm{No}$ as more negatively valenced (consistent with our previous report on 23 of these 27 participants; Alia-Klein et al., 2007). However, there was no main effect of the MAOA genotype; nor was there a Word $\times$ Group interaction for any of the performance and rating variables (all omnibus $F_{\mathrm{S}}<$ 
Table 2

BOLD Response to No Predicts Self-Reported Trait Anger Reactivity

\begin{tabular}{|c|c|c|c|c|c|c|c|}
\hline Region & Size $^{a}$ & $\mathrm{x}$ & $\mathrm{y}$ & $\mathrm{Z}$ & $\mathrm{z}$ & $\begin{array}{l}\text { Anger reactivity: } \\
\text { Pearson } r \text { and } p\end{array}$ & $\begin{array}{l}\text { Regression: } \\
\beta \text { and } p\end{array}$ \\
\hline Left amygdala & 120 & -24 & -3 & -15 & 3.67 & $\begin{array}{l}\mathbf{L}, \mathbf{. 7 8}, . \mathbf{0 0 2} \\
\mathrm{H}, .40, .157 \\
\mathbf{A}, \mathbf{. 5 9}, . \mathbf{0 0 1}\end{array}$ & $\begin{array}{l}\mathbf{L}, . \mathbf{5 7}, . \mathbf{0 3 1} \\
\mathrm{H}, .28, .221 \\
\mathbf{A}, \mathbf{. 5 8}, . \mathbf{0 0 2}\end{array}$ \\
\hline \multirow[t]{3}{*}{ Left thalamus (pulvinar) } & 46 & -6 & -27 & 15 & 3.20 & $\begin{array}{l}\mathbf{L}, \mathbf{. 6 9}, \mathbf{. 0 0 9} \\
\mathbf{H},-. \mathbf{6 5}, .011 \\
\text { A, } .15, .468\end{array}$ & $\begin{array}{l}\mathrm{L}, .34, .171 \\
\mathbf{H},-. \mathbf{6 0}, .019 \\
\mathrm{~A}, .02, .899\end{array}$ \\
\hline & & & & & & & Adjusted $R^{2}$ : \\
\hline & & & & & & & $\begin{array}{l}\mathrm{L}, .62, .003 \\
\mathrm{H}, .41, .021 \\
\mathrm{~A}, .29, .006\end{array}$ \\
\hline
\end{tabular}

Note. These significant responses to No were derived from the No-Up contrast in correlation with anger reactivity in the low-MAOA genotype group (L), the high-MAOA genotype group $(\mathrm{H})$, and the whole sample (A); these responses were validated through comparison of the ROI (regions-of-interest)derived brain responses to No fixation and $U p$ fixation (see Table S4 in the Supplementary Online Results). Correlations at $p<.05$ appear in boldface type. Size $=$ the number of voxels in cluster.

* The test of coincidence of regression lines were significantly different between the MAOA groups $(p<.05)$; the corresponding scatterplots can be found in Figure 2 and in Figure S2 in the Supplementary Online Results.

3.93, $p \mathrm{~s}>0.058$ ). Similarly, no differences emerged between the low- and high-MAOA groups on the STAXI anger reactivity or anger control scales (all $t \mathrm{~s}<1.2, p \mathrm{~s}>0.25$; Table 1). Thus, the gene-brain-behavior relationships reported later cannot be attributed to group differences in trait anger, task performance, or how participants rated the words.

\section{Functional MRI Results}

Gene-brain response to No. As demonstrated previously (Alia-Klein et al., 2007), a one-sample $t$ test with the contrast No- $U p$ revealed that all participants showed relative deactivation in BOLD response to No in the right OFC (Figure S1; Brodmann's Area [BA] 47; Talairach coordinates, $\mathrm{x}=29, \mathrm{y}=31, \mathrm{z}=-4$; extent, 58 voxels, $z$ score $=3.86$ ). This response, however, did not differ between the groups, $t=0.23, p=.81$. For direct group comparison, the same No-Up contrast was used in a two-sample $t$ test as a function of the genotype group; here, low-MAOA participants showed relative BOLD deactivation in the left lateral middle frontal gyrus (BA 9; $\mathrm{x}=-54, \mathrm{y}=9, \mathrm{z}=33 ; 34$ voxels, $\mathrm{z}=3.61$; see Figure 1). Using the extracted ROI in this middle frontal gyrus region, we found a main effect in all participants; for $\mathrm{No}-U p$, $t(26)=-2.3, p=.029$, although it did not reach significance in the SPM2 results described earlier. It is important to note that this effect was significant when the two-sample $t$ test was repeated with $N o$ fixation but not in the $U p$ fixation contrast. Thus, relative deactivation in the left middle frontal gyrus was found specifically to $N o$ in the low-MAOA group, compared with the high-MAOA group, confirming the interaction reported earlier (Figure S5).

Gene-brain and anger reactivity. Further differences between the groups emerged through correlation and regression analyses between the anger regulation variables (i.e., reactivity and control) and the BOLD response to No. Table 2 includes the Pearson correlations between BOLD and trait anger reactivity as a function of the MAOA genotype and in all participants. All participants showed a positive correlation between anger reactivity and BOLD response to No in the left amygdala $(\mathrm{x}=-24, \mathrm{y}=-3, \mathrm{z}=-15$;
120 voxels; $\mathrm{z}$ score $=3.67, p=.001)$. This correlation was driven by the low-MAOA group (see Figure 2 and Figure S2). Moreover, the low-MAOA group showed a similar positive correlation between anger reactivity with the left thalamic (pulvinar) response to No $(\mathrm{x}=-6, \mathrm{y}=-27, \mathrm{z}=15 ; 46$ voxels; $z$ score $=3.20, p=$ .009), whereas the high genotype group showed the opposite correlation. Furthermore, these left thalamic correlations were significantly opposite between the groups; test of coincidence of regression lines, $F(2,23)=3.8, p<.05$. It is also noteworthy that the amygdala and thalamus were positively correlated only in the low-MAOA group (low, $r=.62, p=.023$; high, $r=-0.20, p=$ .498 ; test of coincidence, $F[2,23]=3.1, p<.05$; see Table S2). Multiple regression analyses showed that the combined amygdala and thalamic response to No predicted $62 \%$ of the variance in anger reactivity in the low-MAOA group, adjusted $R^{2}(2,10)=$ $0.62, p=.003$; with an attenuated effect in the high-MAOA group, adjusted $R^{2}(2,11)=0.41, p=.021$.

Gene-brain and anger control. Table 3 includes the Pearson correlations between BOLD and trait anger control as a function of the MAOA genotype and in all participants. All participants showed a positive correlation between BOLD responses to No and anger control in the right lateral OFC (see Figure 3), as previously documented (Alia-Klein et al., 2007). Here, however, this region was not related to anger control differently as a function of the MAOA genotype (for low MAOA, $r=.30, p=.49$; for high MAOA, $r=.20, p=.79$ ). Separate correlation analyses for each genotype group revealed that trait anger control and the BOLD response to No correlated with subcortical regions only in the high MAOA group (see Table 3). Thus, participants with the high genotype who reported more anger control had higher activation in the left insula $(\mathrm{x}=-36, \mathrm{y}=-12, \mathrm{z}=9$; extent, 102 voxels; $z$ score $=3.05, p=.007$ ) and right amygdala-hippocampal formation (AHF; $\mathrm{x}=12, \mathrm{y}=-12, \mathrm{z}=-21$, extent, 110 voxels, $z$ score $=2.78, p=.009)$ in response to $N o$ (see Figure 3 ). Negative correlations (lower activations) in the left $\mathrm{AHF}(\mathrm{x}=-27, \mathrm{y}=-3$, $\mathrm{z}=-18$; extent, 33 voxels; $\mathrm{z}$ score $=3.01$ ) and left anterior 

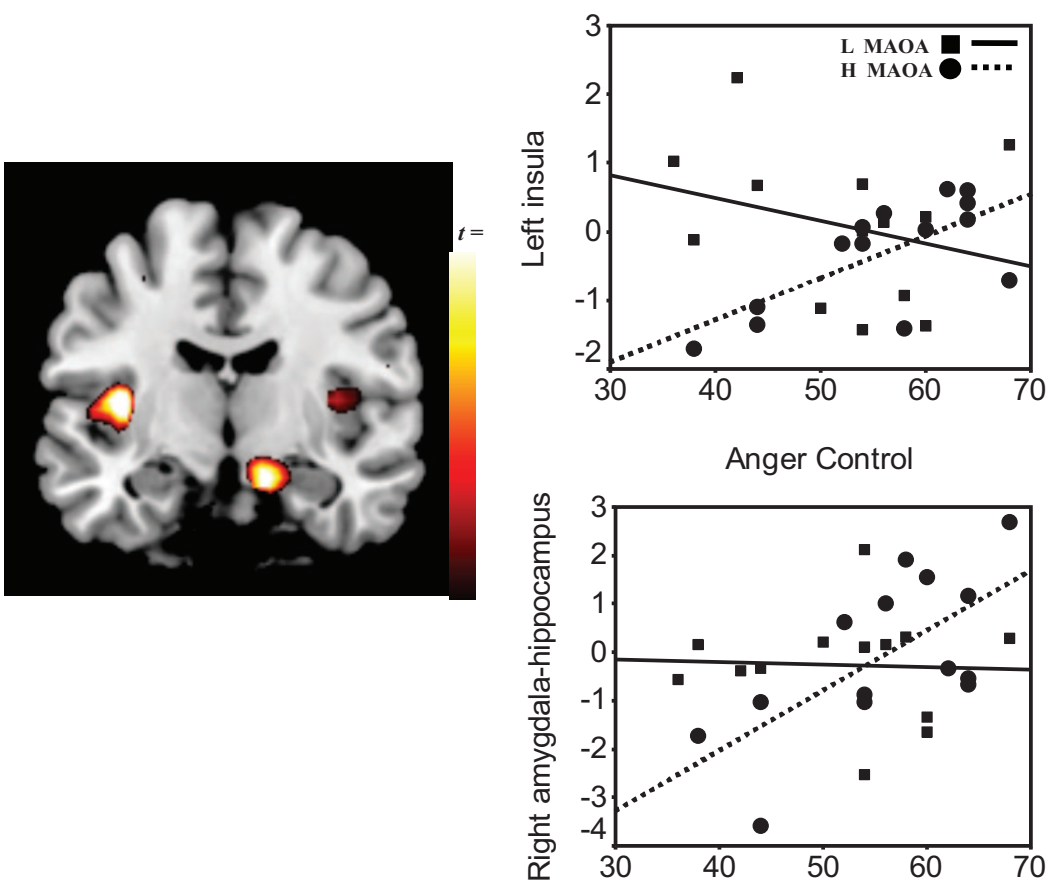

Anger Control

Figure 3. Gene-brain-behavior model in anger control. Left panel: Image of the voxel clusters in positive correlation with self-reported anger control and blood-oxygen-level-dependent (BOLD) response to No in left insula and right amygdala hippocampus formation (AHF). Right panels: Scatterplots of percent BOLD signal change from the left insula (top right panel: low MAOA, $R^{2}=0.08, p=.344$; vs. high MAOA, $R^{2}=0.48, p=$ .007 ) and right AHF (bottom panel: low MAOA, $R^{2}=0.006, p=.897$; vs. high MAOA, $R^{2}=0.45, p=.009$ ). This figure was derived from the $N o-U p$ contrast and validated through comparison of the brain responses, derived from regions of interest, to No fixation and $U p$ fixation (see Table S5 in the Supplementary Online Results). Squares and solid regression line indicate low (L) MAOA; circles and dotted line indicate high $(\mathrm{H})$ MAOA.

thalamus $(\mathrm{x}=-15, \mathrm{y}=-6, \mathrm{z}=6$; extent, 9 voxels; $\mathrm{z}$ score $=$ 2.99 ) to No were also driven by the high MAOA group, although they were observed in all participants (see Figure 4). Furthermore, the left insula, right thalamus, and right AHF correlations with anger control were significantly opposite between the groups (test of coincidence of regression lines; left insula, $F(2,23)=3.4$, right $\mathrm{AHF}=3.0$, right thalamus, $F(2,23)=3.7, p<.05$. It is also noteworthy that the left insula and left thalamus were correlated with each other only in the high MAOA group (low MAOA, $r=$ $.46, p=.111$; high MAOA, $r=-0.60, p=.021)$; test of coincidence, $F(2,23)=3.1, p<.05$ (see Table S3). Multiple regression analyses showed that, combined, these brain regions explained $73 \%$ of the variance in anger control in the high-MAOA group, adjusted $R^{2}(2,11)=0.73, p=.005$, but did not predict any significant variance in the low-MAOA group, adjusted $R^{2}(2,11)=$ $0.02, p=.500$.

\section{Discussion}

This study highlights the brain circuits where response to prohibitive language (e.g., an emphatically expressed $N o$ !) is differentially associated with trait anger regulation as a function of the MAOA genotype, a known genetic risk factor for aggressive behavior. As predicted, these healthy nonsmoking men did not differ on any of the task-related behavioral measures or on selfreported trait anger. Nevertheless, compared with the high-MAOA group, the low-MAOA participants responded to No with relatively deactivated BOLD signal in the left middle frontal gyrus. Furthermore, the low-MAOA participants who reported higher anger reactivity had increased left amygdala and thalamus response to No. In contrast, higher self-reported anger control in the high-MAOA group correlated with more left insula and right AHF activation and suppressed bilateral thalamus and left AHF activation to $\mathrm{No}$.

\section{Involvement of the OFC in BOLD Response to No}

Consistent with our previous study (Alia-Klein et al., 2007) brain response to $\mathrm{No}$ (word main effect) involved a negative signal in the right lateral OFC in all study participants. Similarly, in this and our previous study, participants who reported high anger control showed increased activation to $N o$ in a contiguous region of the right lateral OFC. These responses, however, did not differ as a function of the MAOA genotype; an increased sample size for this particular genotype comparison may be needed to ensure that the lack of an effect is not due to limited statistical power. 
Table 3

BOLD Response to No Predicts Self-Reported Trait Anger Control

\begin{tabular}{|c|c|c|c|c|c|c|c|}
\hline Region & Size & $\mathrm{x}$ & $\mathrm{y}$ & $\mathrm{z}$ & $\mathrm{Z}$ & $\begin{array}{l}\text { Anger control: } \\
\text { Pearson } r \text { and } p\end{array}$ & $\begin{array}{l}\text { Regression: } \\
\beta \text { and } p\end{array}$ \\
\hline Left insula & 102 & -36 & -12 & 9 & 3.05 & $\begin{array}{l}\mathrm{L},-.29, .344 \\
\mathbf{H}, \mathbf{. 6 9 , . 0 0 7 *} \\
\mathrm{A},-.07, .731\end{array}$ & $\begin{array}{l}\mathrm{L}, .11, .785 \\
\mathrm{H}, .43, .057 \\
\mathrm{~A}, .00, .987\end{array}$ \\
\hline Left AHF & 140 & -30 & -3 & -24 & 2.81 & $\begin{array}{l}\mathrm{L},-.55, .053 \\
\mathbf{H},-\mathbf{6 9}, .006 \\
\mathbf{A},-\mathbf{5 8}, \mathbf{. 0 0 2}\end{array}$ & $\begin{array}{l}\mathrm{L},-.60, .134 \\
\mathrm{H}, .33, .139 \\
\mathbf{A},-\mathbf{4 4}, . \mathbf{0 2 4}\end{array}$ \\
\hline Right AHF & 110 & 12 & -12 & -21 & 2.78 & $\begin{array}{l}\text { L, }-.04, .897 \\
\mathbf{H}, . \mathbf{6 7}, . \mathbf{0 0 9} \\
\text { A, } .38, .055\end{array}$ & $\begin{array}{l}\text { L, .16, .669 } \\
\mathbf{H},-. \mathbf{4 0}, .047 \\
\text { A, .26, .143 }\end{array}$ \\
\hline Left thalamus (anterior) & 235 & -15 & -6 & 6 & 3.27 & $\begin{array}{l}\mathrm{L},-.30, .326 \\
\mathbf{H},-. \mathbf{7 6}, .001 \\
\mathbf{A},-\mathbf{4 4 , . 0 2 0}\end{array}$ & $\begin{array}{l}\mathrm{L},-.36, .395 \\
\mathrm{H},-.14, .654 \\
\mathrm{~A},-0.24, .300\end{array}$ \\
\hline \multirow[t]{3}{*}{ Right thalamus (anterior) } & 376 & 12 & -9 & 9 & 2.93 & $\begin{array}{l}\mathrm{L}, .08, .782 \\
\mathbf{H},-\mathbf{. 6 8}, .008^{*} \\
\mathrm{~A},-.28, .163\end{array}$ & $\begin{array}{l}\mathrm{L}, .12, .760 \\
\mathrm{H}, .09, .757 \\
\mathrm{~A}, .06, .805\end{array}$ \\
\hline & & & & & & & Adjusted $\mathrm{R}^{2}$ \\
\hline & & & & & & & $\begin{array}{l}\mathrm{L},-.02, .500 \\
\mathbf{H}, \mathbf{. 7 3}, \mathbf{. 0 0 5} \\
\mathbf{A}, \mathbf{. 3 0}, \mathbf{. 0 2 0}\end{array}$ \\
\hline
\end{tabular}

Note. Responses to No were derived from the No-Up contrast in correlation with anger control in the low-MAOA genotype group (L), the high-MAOA genotype group $(\mathrm{H})$, and the whole sample (A); these responses were validated through comparison of the ROI (regions-of-interest)-derived brain responses to No fixation and $U p$ fixation (see Table S5 in Supplementary Online Results). Correlations at $p<.05$ appear in boldface type. Size $=$ number of voxels in cluster; $\mathrm{AHF}=$ amygdala hippocampus formation.

${ }^{*}$ The coincidence of the regression lines were significantly different between the MAOA groups, $p<.05$; the corresponding scatterplots can be found in Figures 3 and 4 and in Figures S3 and S4 in the Supplementary Online Results.

\section{Gene-Brain Interaction: Reduced Middle Frontal Response to No}

An interaction between word and group was observed in the left middle frontal gyrus (BA 9). This region was relatively deactivated to $N o-U p$ in carriers of the low-MAOA genotype, whereas it was activated in carriers of the high-MAOA genotype (driven by No but not by $U p$; see Figure 1). Given the middle frontal gyrus involvement in resolving cognitive conflict (Fan, Flombaum, et al., 2003; Fan, Fossella, et al., 2003), this interaction suggests that the high-MAOA groups may be more engaged in conflict resolution when hearing No than carriers of the low-MAOA genotype. This interpretation is supported by our present behavioral findings of slower RT to No versus $U p$ in this fMRI task in all study participants, which is consistent with our previous suggestion that pressing a button while hearing $N o$ (vs. $U p$ ) creates conflict (Alia-Klein et al., 2007). Nevertheless, this speculation awaits future studies using standard response inhibition tasks (e.g., go/no-go) as cognitive platforms with prohibitive language. In addition, the utterance No can be heard or expressed and these are distinct processes. The task in this study involved hearing the word No while saying it subvocally. It would therefore be important to study the neural correlates of saying No and hearing No in separate conditions.

\section{Gene-Brain-Behavior Interaction in Anger Reactivity}

Increased anger reactivity in the low-MAOA participants was associated with left amygdala and posterior thalamic (pulvinar) activity in response to No. In contrast, the respective associations for the high-MAOA group did not reach significance (left amyg- dala) or showed the opposite direction (thalamus-pulvinar; see Figure 2). Pulvinar thalamic activation in neuroimaging studies is observed under flanker conditions, interpreted to contribute to filtering irrelevant information (LaBerge \& Buchsbaum, 1990; Rafal \& Posner, 1987). Primate studies suggest that the pulvinar has a role in directing attention or intention through eye and limb goal-directed movement (Grieve, Acuna, \& Cudeiro, 2000). However, more recent studies have provided evidence that the pulvinar is involved in the unconscious recognition of fear (Ward, Calder, Parker, \& Arend, 2007). Although these findings have been specifically related to the visual perception of fearful faces, future studies will determine whether they are also involved on processing of visual language that may be conditioned to fear responses (Skuse, 2006). The amygdala findings are similar to earlier studies by Meyer-Lindenberg and colleagues (2006), who reported amygdala reactivity to emotion paradigms (and reduced gray matter in the bilateral amygdala) in low-, compared with high-MAOA groups. Imaging studies have also noted amygdala activation with exposure to angry faces (Whalen et al., 2001) and in the retrieval of conceptual knowledge from words (Adolphs, 2000). The amygdala is particularly relevant to MAOA enzymatic activity. The sex hormone receptors are prominently expressed in amygdala and able to influence monoamine metabolism by regulating MAOA (Gundlah, Lu, \& Bethea, 2002). Mouse knockouts for MAOA show enhanced amygdala-dependent emotional learning but not motor learning (Kim et al., 1997), and male mice exhibit dramatically increased aggressive behavior (Cases et al., 1995). In humans, a genetic locus at Xp11.3, MAOA gene, appears to play a key role in amygdala development (Good et al., 2003). 

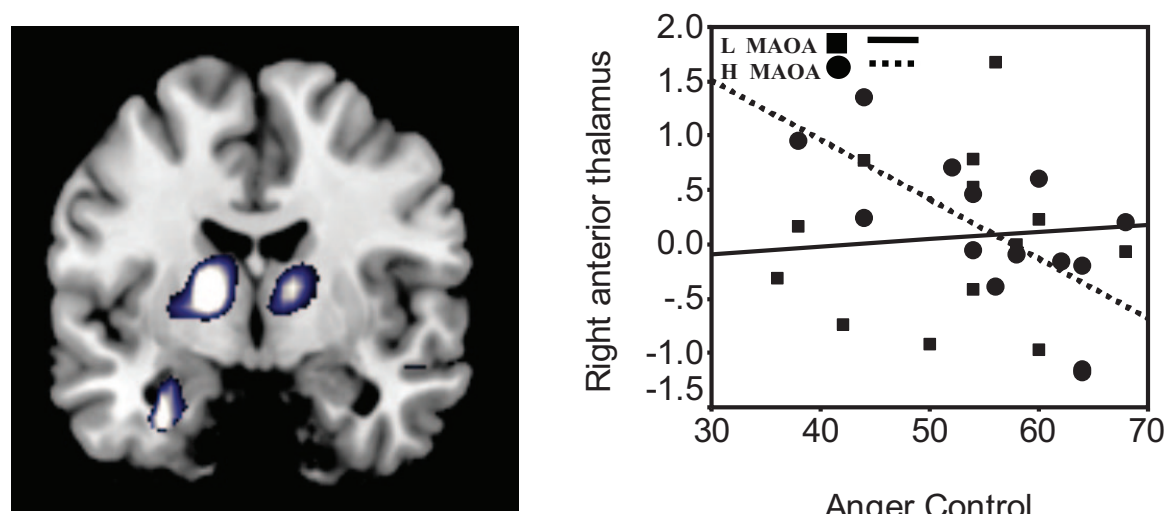

\section{Anger Control}
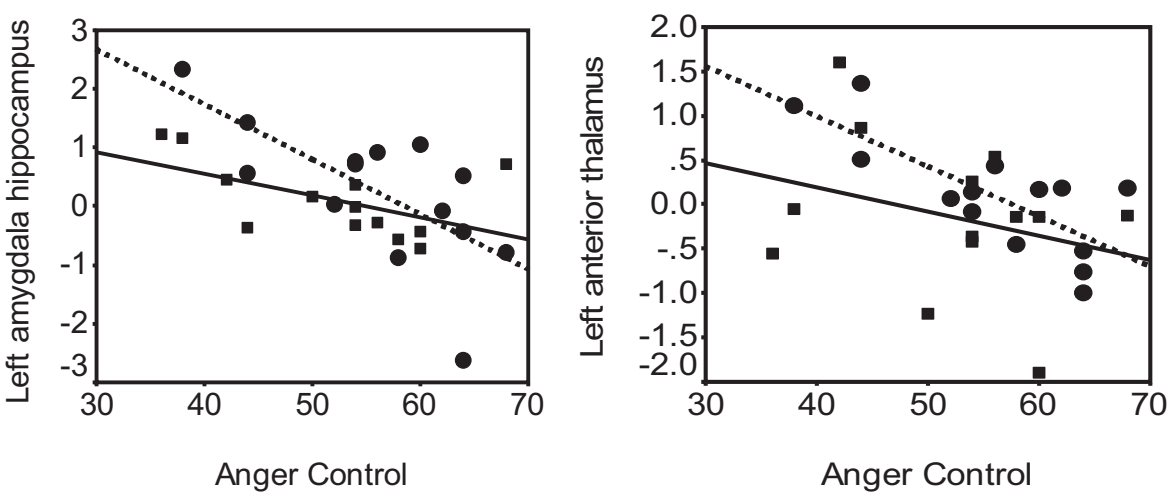

Figure 4. Gene-brain-behavior model in anger control. Top left panel: Image of the voxel clusters in negative correlation with self-reported anger control and blood-oxygen-level-dependent (BOLD) response to No in bilateral thalamus and left amygdala-hippocampal formation (AHF). The corresponding scatterplots represent of percent BOLD signal change in left AHF (low MAOA, $R^{2}=0.30, p=.053$; vs. high MAOA, $R^{2}=0.48, p=$ .009 ), left anterior thalamus (low MAOA, $R^{2}=0.09, p=.326$; vs. high MAOA, $R^{2}=0.58, p=.001$ ), and right anterior thalamus (low MAOA, $R^{2}=0.00, p=.782$; vs. high MAOA, $R^{2}=0.46, p=.008$ ). This figure was derived from the $N o-U p$ contrast and validated through comparison of brain responses, derived from regions of interest, to No fixation and $U p$ fixation (see Table S5 in the Supplementary Online Results). Squares and solid regression line indicate low (L) MAOA; circles and dotted line indicate high (H) MAOA.

The significant correlations between the amygdala and the thalamus are consistent with the known neuroanatomical connections between the pulvinar with the amygdala (Jones \& Burton, 1976). Thus, in the context of the amygdala and the thalamic-pulvinar response to No in association with anger reactivity, several interpretations can be made. Possibly, attention to emotion is mediated by the amygdala, whereas its connections to the thalamus may mediate these attentional effects in preparation for action (Kensinger \& Corkin, 2004). However, it is more likely that it reflects the activation of the amygdala through the thalamus in a way that will not require attentional engagement and cortical activation (LeDoux, 1996). This "low road" is described as a distinct thalamus-pulvinar pathway to the amygdala that is automatic and precludes conscious attention (Öhman, 2005). It is noteworthy that the amygdala and thalamus-pulvinar responses to No were correlated in the low-MAOA group only; together, these regions' responses to $N o$ explained a higher variance of trait anger reactivity in the low- versus the high-MAOA group (62\% vs. $41 \%$, respectively). Thus, anger reactivity in the low-MAOA group may be characterized by automatic and nonexecutive response to $\mathrm{No}$.

\section{Gene-Brain-Behavior Interaction in Anger Control}

The higher the anger control, the more left insula and right AHF response to $\mathrm{No}$ as driven by carriers of the high-MAOA genotype; in contrast, the respective correlations for the low-MAOA group were not significant (see Figure 3). The insula generates autonomic responses that are important for mapping visceral states and bringing interoceptive signals to conscious awareness (Bechara \& Naqvi, 2004), and ultimately the insula, has a core involvement in decision making (Bechara \& Damasio, 2002). The AHF is involved in learning and memory, reflecting the influence of previous affective experiences, and is implicated in the stress response to aversive conditioned stimuli including fear (Alvarez, Biggs, Chen, Pine, \& Grillon, 2008; Birbaumer et al., 1998; Strauss et al., 2005). Activation of these two regions to No in high-MAOA participants reporting increased anger control may indicate that these participants engaged somatic-emotional learning when exposed to prohibitive language. In addition, the higher the anger control in carriers of the high-MAOA genotype, the lower the response was to No in the left $\mathrm{AHF}$ and bilateral anterior thalamus 
(see Figure 4). These correlations may reflect neural suppression of regions that would otherwise be reactive to emotional stimuli (see Table 3).

Together, the insula, AHF, and thalamus response to No explained $73 \%$ of anger control in the high-MAOA participants (and none in the low-MAOA genotype; see Table 3). These findings may suggest that carriers of the high-MAOA genotype could be utilizing differential strategies, encompassing advantageous suppression of emotionally reactive regions, to control anger to emotionally negative stimuli. However, this interpretation is provisional and it requires further testing. In particular, studies targeting higher-order learning behavior as a result of hearing $N o$ are needed.

\section{Neural Mechanisms of Anger Regulation as Endophenotypes of Violence}

Our results support a growing body of knowledge on the intermediate phenotypes predicting risk for aggressive behavior (Buckholtz et al., 2007) while extending this knowledge to anger regulation as a core aspect of risk for aggression. Risk for aggressive behavior, however, does not always translate to overt violent behavior. Indeed, although conflicting results exist (Haberstick et al., 2005), several independent studies do not show a significant difference on trait aggression as a function of the MAOA genotype alone; instead, these studies show that having the low-MAOA genotype predicts increased aggression and violence only in a minority of cases with history of severe childhood maltreatment, thus demonstrating a gene-environment interaction (Caspi et al., 2002; Foley et al., 2004; Huang et al., 2004; Kim-Cohen et al., 2006; Widom \& Brzustowicz, 2006). Indeed, it is not reasonable to assume that individuals carrying the low-MAOA alleles, making up approximately $40 \%$ of the male population (Sabol et al., 1998), regularly exhibit poor anger regulation or aggressive behavior.

As with trait behavior, brain MAO A enzymatic activity (Fowler et al., 2007), as well as brain glucose metabolism at a resting baseline (Alia-Klein, Kriplani, et al., 2008), does not reveal differences between low- and high-MAOA genotype groups. Nevertheless, low brain MAO A activity in vivo is associated with relatively high self-reported aggression in healthy nonsmoking men, independent of the MAOA genotype (Alia-Klein, Goldstein, et al., 2008). Thus, in addition to interaction with the environment, the risk for aggression may be mediated by catabolism of monoamines by brain MAO A similarly expressed across genotype groups.

Because brain MAO A activity is not modulated by the MAOA genotype in this sample (Fowler et al., 2007) and because trait aggression is most often not directly modulated by the MAOA genotype, it remains unknown how-or rather, when-the low versus high genotype exerts its influence on susceptibility to violent conduct. Current thinking points to sensitive periods during prenatal development and early childhood that affect the availability of MAO A and MAO B enzymatic activity in the developing nervous system (Fowler et al., 2004; Whitaker-Azmitia, Zhang, \& Clarke, 1994). These MAO-mediated changes possibly trigger compensatory mechanisms that are in place to ensure a dynamic balance of monoamine function through adulthood (Mejia, Ervin, Baker, \& Palmour, 2002).
In conclusion, our results suggest that carriers of the lowMAOA genotype may have developed stable stimulus-response biases to prohibitive language that are related to enhanced activity in brain circuits that modulate anger reactivity. Conversely, carriers of the high-MAOA genotype may have developed stimulusresponse biases to prohibitive language that are related to enhanced engagement in circuits that modulate anger control. In general, it is becoming clear that multilayered gene-brainbehavior models will help predict aberrant aggression in specific populations and under specific conditions. Therefore, it remains to translate these endophenotypic models from basic human studies to populations of individuals with chronic anger and episodes of violent behavior as observed, for example, in social-relational situations that involve domestic abuse.

\section{References}

Adolphs, R. (2000). Emotion, recognition, and the human amygdala. In J. P. Aggeleton (Ed.), The amygdala (2nd ed., pp. 587-622). New York: Oxford University Press.

Alia-Klein, N., Goldstein, R. Z., Kriplani, A., Logan, J., Tomasi, D., Williams, B., et al. (2008). Brain monoamine oxidase A activity predicts trait aggression. Journal of Neuroscience, 28, 5099-5104.

Alia-Klein, N., Goldstein, R. Z., Tomasi, D., Zhang, L., Fagin-Jones, S., Telang, F., et al. (2007). What is in a word? No versus Yes differentially engage the lateral orbitofrontal cortex. Emotion, 7, 649-659.

Alia-Klein, N., Kriplani, A., Pradhan, K., Ma, J. Y., Logan, J., Williams, B., et al. (2008). The MAOA genotype does not modulate resting brain metabolism in adults. Psychiatry Research, 164, 73-76.

Alvarez, R. P., Biggs, A., Chen, G., Pine, D. S., Grillon, C. (2008). Contextual fear conditioning in humans: Cortical-hippocampal and amygdala contributions. Journal of Neuroscience, 28, 6211-6219.

Ashburner, J., Neelin, P., Collins, D. L., Evans, A., \& Friston, K. (1997). Incorporating prior knowledge into image registration. Neuroimage, 6 , $344-352$.

Barratt, E. S., Stanford, M. S., Dowdy, L., Liebman, M. J., \& Kent, T. A. (1999). Impulsive and premeditated aggression: A factor analysis of self-reported acts. Psychiatry Research, 86, 163-173.

Bechara, A. B., \& Damasio, H. (2002). Decision-making and addiction (Part I): Impaired activation of somatic states in substance dependent individuals when pondering decisions with negative future consequences. Neuropsychologia, 40, 1675-1689.

Bechara, A., \& Naqvi, N. (2004). Listening to your heart: Interoceptive awareness as a gateway to feeling. Nature Neuroscience, 7, 102-103.

Beck, A. T., Steer, R. A., \& Brown, G. K. (1996). Beck Depression Inventory manual (2nd ed.). San Antonio, TX: The Psychological Corporation.

Birbaumer, N., Grodd, W., Diedrich, O., Klose, U., Erb, M., Lotze, M., et al. (1998). fMRI reveals amygdala activation to human faces in social phobics. Neuroreport, 9, 1223-1226.

Bloom, L. (1973). One word at a time. The Hague, the Netherlands: Mouton.

Brunner, H. G., Nelen, M., Breakefield, X. O., Ropers, H. H., \& van Oost, B. A. (1993). Abnormal behavior associated with a point mutation in the structural gene for monoamine oxidase A. Science, 262, 578-580.

Buckholtz, J. W., Callicott, J. H., Kolachana, B., Hariri, A. R., Goldberg, T. E., Genderson, M., et al. (2007). Genetic variation in MAOA modulates ventromedial prefrontal circuitry mediating individual differences in human personality. Molecular Psychiatry, 13, 313-324.

Buonocore, M. H., \& Gao, L. (1997). Ghost artifact reduction for echo planar imaging using image phase correction. Magnetic Resonance in Medicine, 38, 89-100.

Cases, O., Seif, I., Grimsby, J., Gaspar, P., Chen, K., Pournin, S., et al. 
(1995). Aggressive behavior and altered amounts of brain serotonin and norepinephrine in mice lacking MAOA. Science, 268, 1763-1766.

Caspi, A., McClay, J., Moffitt, T. E., Mill, J., Martin, J., Craig, I. W., et al. (2002). Role of genotype in the cycle of violence in maltreated children. Science, 297, 851-854.

Davidson, R. J., Putnam, K. M., \& Larson, C., L. (2000). Dysfunction in the neural circuitry of emotion regulation-A possible prelude to violence. Science, 289, 591-594.

Dimberg, U. (1986). Facial reactions to fear-relevant and fear-irrelevant stimuli. Biological Psychology, 23, 153-161.

Dougherty, D. D., Rauch, S. L., Deckersbach, T., Marci, C., Loh, R., Shin, L. M., et al. (2004). Ventromedial prefrontal cortex and amygdala dysfunction during an anger induction positron emission tomography study in patients with major depressive disorder with anger attacks. Archives of General Psychiatry, 61, 795-804.

Driscoll, H., Zinkivskay, A., Evans, K., \& Campbell, A. (2006). Gender differences in social representations of aggression: The phenomenological experience of differences in inhibitory control? British Journal of Psychology, 97, 139-153.

Eisenberger, N. I., Way, B. M., Taylor, S. E., Welch, W. T., \& Lieberman, M. D. (2007). Understanding genetic risk for aggression: Clues from the brain's response to social exclusion. Biological Psychiatry, 61, 11001108.

Fan, J., Flombaum, J. I., McCandliss, B. D., Thomas, K. M., \& Posner, M. I. (2003). Cognitive and brain consequences of conflict. Neuroimage, $18,42-57$.

Fan, J., Fossella, J., Sommer, T., Wu, Y., \& Posner, M. I. (2003). Mapping the genetic variation of executive attention onto brain activity. Proceedings of the National Academy of Science USA, 100, 7406-7411.

Foley, D. L., Eaves, L. J., Wormley, B., Silberg, J. L., Maes, H. H., Kuhn, J., et al. (2004). Childhood adversity, monoamine oxidase a genotype, and risk for conduct disorder. Archives of General Psychiatry, 61, $738-744$.

Fowler, J. S., Alia-Klein, N., Kriplani, A., Logan, J., Williams, B., Zhu, W., et al. (2007). Evidence that brain MAO A activity does not correspond to MAO A genotype in healthy male subjects. Biological Psychiatry, 62, 355-358.

Fowler, J. S., Logan, J., Wang, G. J., Volkow, N. D., Telang, F., Ding, Y. S., et al. (2004). Comparison of the binding of the irreversible monoamine oxidase tracers, [(11)C $]$ clorgyline and [(11)C $]$-deprenyl in brain and peripheral organs in humans. Nuclear Medicine Biology, 31, 313-319.

Fowler, J. S., MacGregor, R. R., Wolf, A. P., Arnett, C. D., Dewey, S. L., Schlyer, D., et al. (1987). Mapping human brain monoamine oxidase A and B with 11C-labeled suicide inactivators and PET. Science, 235, 481-485.

Freeman, B., Smith, N., Curtis, C., Huckett, L., Mill, J., \& Craig, I. W. (2003). DNA from buccal swabs recruited by mail: Evaluation of storage effects on long-term stability and suitability for multiplex polymerase chain reaction genotyping. Behavioral Genetics, 33, 67-72.

Friston, K. J., Holmes, A. P., Worsley, K. J., Poline, J. B., Frith, C. D., \& Frackowiak, R. S. (1995). Statistical parametric maps in functional imaging: A general approach. Human Brain Mapping, 2, 189-210.

Glantz, S. A. (2005). Primer of biostatistics (6th ed.). McGraw-Hill.

Good, C. D., Lawrence, K., Thomas, N. S., Price, C. J., Ashburner, J., Friston, K. J., et al. (2003). Dosage-sensitive X-linked locus influences the development of amygdala and orbitofrontal cortex, and fear recognition in humans. Brain, 126, 2431-2446.

Gopnik, A. (1984). The acquisition of gone and the development of the object concept. Journal Child Language, 11, 273-292.

Gopnik, A., \& Meltzoff, A. (1985). From people, to plans, to objects: Changes in the meaning of early words and their relation to cognitive development. Journal of Pragmatics, 9, 495-512.

Gottesman, I. I., \& Gould, T. D. (2003). The endophenotype concept in psychiatry: Etymology and strategic intentions. American Journal Psychiatry, 160, 636-645.

Grieve, K. L., Acuna, C., \& Cudeiro, J. (2000). The primate pulvinar nuclei: Vision and action. Trends in Neuroscience, 23, 35-39.

Gundlah, C., Lu, N. Z., \& Bethea, C. L. (2002). Ovarian steroid regulation of monoamine oxidase-A and -B mRNAs in the macaque dorsal raphe and hypothalamic nuclei. Psychopharmacology (Berl), 160, 271-282.

Haberstick, B. C., Lessem, J. M., Hopfer, C. J., Smolen, A., Ehringer, M. A., Timberlake, D., et al (2005). Monoamine oxidase A (MAOA) and antisocial behaviors in the presence of childhood and adolescent maltreatment. American Journal of Medical Genetics, Part B: Neuropsychiatric Genetics, 135, 59-64.

Harmon-Jones, E. (2004). Contributions from research on anger and $\operatorname{cog}$ nitive dissonance to understanding the motivational functions of asymmetrical frontal brain activity. Biological Psychology, 67, 51-76.

Hewig, J., Hagemann, D., Seifert, J., Naumann, E., \& Bartussek, D. (2004). On the selective relation of frontal cortical asymmetry and anger-out versus anger control. Journal of Personality and Social Psychology, 87, 926-939.

Hollingshead, A. B. (1975). Four-factor index of social status. Department of Sociology, Yale University.

Huang, Y. Y., Cate, S. P., Battistuzzi, C., Oquendo, M. A., Brent, D., \& Mann, J. J. (2004). An association between a functional polymorphism in the monoamine oxidase a gene promoter, impulsive traits and early abuse experiences. Neuropsychopharmacology, 29(8), 1498-1505.

Jacob, C. P., Muller, J., Schmidt, M., Hohenberger, K., Gutknecht, L., Reif, A., et al. (2005). Cluster B personality disorders are associated with allelic variation of monoamine oxidase A activity. Neuropsychopharmacology, 30, 1711-1718.

Jones, E. G., \& Burton, H. (1976). A projection from the medial pulvinar to the amygdala in primates. Brain Research, 104, 142-147.

Kensinger, E. A., \& Corkin, S. (2004). Two routes to emotional memory: Distinct neural processes for valence and arousal. Proceedings of the National Academy of Science USA, 101, 3310-3315.

Kim, J. J., Shih, J. C., Chen, K., Chen, L., Bao, S., Maren, S., et al. (1997). Selective enhancement of emotional, but not motor, learning in monoamine oxidase A-deficient mice. Proceedings of the National Academy of Science USA, 94, 5929-5933.

Kim-Cohen, J., Caspi, A., Taylor, A., Williams, B., Newcombe, R., Craig, I. W., et al. (2006). MAOA, maltreatment, and gene-environment interaction predicting children's mental health: New evidence and a metaanalysis. Molecular Psychiatry, 11, 903-913.

Kochanska, G., \& Knaack, A. (2003). Effortful control as a personality characteristic of young children: Antecedents, correlates, and consequences. Journal of Personality, 71, 1087-1112.

Kučera, \& Francis. (1967). Computational analysis of present-day American English. Providence, RI: Brown University Press.

LaBerge, D., \& Buchsbaum, M. S. (1990). Positron emission tomographic measurements of pulvinar activity during an attention task. Journal of Neuroscience, 10, 613-619.

LeDoux, J. E. (1996). The emotional brain. Simon \& Schuster, New York. Manuck, S. B., Flory, J. D., Ferrell, R. E., Mann, J. J., \& Muldoon, M. F. (2000). A regulatory polymorphism of the monoamine oxidase A gene may be associated with variability in aggression, impulsivity, and central nervous system serotonergic responsivity. Psychiatry Research, 95, $9-23$.

Mejia, J. M., Ervin, F. R., Baker, G. B., Palmour, R. M. (2002). Monoamine oxidase inhibition during brain development induces pathological aggressive behavior in mice. Biological Psychiatry, 52, 811-821.

Meyer-Lindenberg, A., Buckholtz, J. W., Kolachana, B., Hariri, A. R., Pezawas, L., Blasi, G., et al. (2006). Neural mechanisms of genetic risk for impulsivity and violence in humans. Proceedings of the National Academy of Sciences USA, 103, 6269-6274.

Meyer-Lindenberg, A., \& Weinberger, D. R. (2006). Intermediate pheno- 
types and genetic mechanisms of psychiatric disorders. Nature Review Neuroscience, 7, 818-827.

Murphy, F. C., Nimmo-Smith, I., \& Lawrence, A. D. (2003). Functional neuroanatomy of emotions: A meta-analysis. Cognitive Affective Behavior Neuroscience, 3, 207-233.

Öhman, A. (2005). The role of the amygdala in human fear: Automatic detection of threat. Psychoneuroendocrinology, 30, 953-958.

Oldfield, R. C. (1971). The assessment and analysis of handedness: The Edinburgh Handedness Inventory. Neuropsychologia, 9, 97-113.

Passamonti, L., Fera, F., Magariello, A., Cerasa, A., Gioia, M. C., Muglia, M., et al. (2006). Monoamine oxidase-A genetic variations influence brain activity associated with inhibitory control: New insight into the neural correlates of impulsivity. Biological Psychiatry, 59, 334-340.

Rafal, R. D., \& Posner, M. I. (1987). Deficits in human visual spatial attention following thalamic lesions. Proceeding of the National Academy of Science USA, 84, 7349-7353.

Sabol, S. Z., Hu, S., \& Hamer, D. (1998). A functional polymorphism in the monoamine oxidase A gene promoter. Human Genetics, 103, 273279

Scarpa, A., \& Raine, A. (1997). Psychophysiology of anger and violent behavior. Psychiatric Clinics of North America, 20, 375-394.

Shih, J. C., Chen, K., \& Ridd, M. J. (1999). Monoamine oxidase: From genes to behavior. Annals Review Neuroscience, 22, 197-217.

Skuse, D. (2006). Genetic influences on the neural basis of social cognition. Philosophical Transactions of the Royal Society Britain, 361, 2129-2141.

Spielberger, C. (1988). Manual for the State-Trait Anger Expression Inventory. Odessa, FL: Psychological Assessment Resources.

Stevens, J. (1992). Applied multivariate statistics for the social sciences (2nd ed.). Mahwah, NJ: Erlbaum.
Strauss, M. M., Makris, N., Aharon, I., Vangel, M. G., Goodman, J., Kennedy, D. N., et al. (2005). fMRI of sensitization to angry faces. Neuroimage, 26, 389-413.

Talairach, J., \& Tournoux, P. (1988). Co-planar stereotaxic atlas of the human brain. New York: Thieme Medical Publishers.

Ward, R., Calder, A. J., Parker, M., \& Arend, I. (2007). Emotion recognition following human pulvinar damage. Neuropsychologia, 45, 1973 1978.

Wechsler, D. (1999). Wechsler abbreviated scale of intelligence: San Antonio, TX: Psychological Corporation.

Whalen, P. J., Shin, L. M., McInerney, S. C., Fischer, H., Wright, C. I., \& Rauch, S. L. (2001). A functional MRI study of human amygdala responses to facial expressions of fear versus anger. Emotion, 1, 70-83.

Whitaker-Azmitia, P. M., Zhang, X., \& Clarke, C. (1994). Effects of gestational exposure to monoamine oxidase inhibitors in rats: Preliminary behavioral and neurochemical studies. Neuropsychopharmacology, $11,125-132$.

Widom, C. S., \& Brzustowicz, L. M. (2006). MAOA and the "cycle of violence": Childhood abuse and neglect, MAOA genotype, and risk for violent and antisocial behavior. Biological Psychiatry, 60, 684-689.

Wilkinson, G. (1993). The Wide Range Achievement Test 3-Administration manual. Wilmington, DE: Wide Range.

Worsley, K. J., Marrett, S., Neelin, P., Vandal, A. C., Friston, K. J., \& Evans, A. C. (1996). A unified statistical approach for determining significant signals in images of cerebral activation. Human Brain Mapping, 4, 58-73.

Received October 22, 2007

Revision received January 22, 2009

Accepted January 22, 2009 